\title{
Sex differences in lateralization for spatial abilities
}

\author{
SHERYL A. KINGSBERG, RICHARD C. LABARBA, and CLINT A. BOWERS \\ University of South Florida, Tampa, Florida
}

\begin{abstract}
Sex differences in cerebral lateralization for spatial abilities were studied using a dual-task paradigm. In the first phase of this experiment, both males and females were found to display similar cerebral lateralization patterns for manipulospatial processing of block designs across two levels of difficulty. There was no evidence of laterality effects in right-hand or left-hand tapping, but analysis of trade-off effects revealed that both males and females took significantly longer to solve the block designs when simultaneously tapping with the right hand and manipulating the block designs with the left hand. No specific lateralization patterns were found either within or between sexes. In Phase 2 of this study, it was observed that females may use a verbal mediation strategy in manipulospatial processing insofar as interference effects were found during concurrent vocalization and manipulospatial processing in females.
\end{abstract}

Investigations of sex differences in the cerebral lateralization of visuospatial abilities continue to produce contradictory and confusing results. Although the evidence for differential performance between males and females on spatial tasks is unequivocal, the origins of male advantage in spatial processing remain obscure. The major prevailing hypothesis for these observed sex differences is that males and females possess differential patterns of brain organization for visuospatial processing (Buffery \& Gray, 1972; Levy, 1972). Although there is now a large body of evidence suggesting that the right cerebral hemisphere is largely involved in the control and processing of visuospatial tasks, the evidence for sex differences in this asymmetrical pattern of cerebral organization is inconsistent.

Among the behavioral approaches to this question in normal populations, the dual-task paradigm (Kinsbourne, 1981) has only rarely been used. Wolff, Hurwitz, and Moss (1977) were the first to attempt to identify sex differences in cerebral lateralization for a spatial ability (rhythm) by this concurrent task procedure. They found that females are more right lateralized than males for this kind of spatial task. Dalby (1980) used a dual-task paradigm in which subjects were required to sequentially tap the four fingers of each hand while simultaneously performing one of four spatial or verbal tasks.

On a progressive matrices task, both sexes displayed right-hemisphere lateralization, but the results on the Space Relations test from the Differential Aptitude Test indicated right lateralization for males and bilateralization for females. Hellige and Longstreth (1981) found no evidence of sex differences in cerebral organization on

This paper is based on a thesis submitted by the first author to the Department of Psychology, University of South Florida, in partial fulfillment of the requirements for the master's degree in psychology. Requests for reprints should be directed to S. A. Kingsberg, Department of Psychology, University of South Florida, Tampa, FL 33620. block design performance and concurrent finger tapping. They reported a significant right laterality effect for both sexes, but only when the block designs were solved manually. The mental solution condition revealed no such behavioral asymmetry pattern. In an extension of the Hellige and Longstreth study, Kee, Bathhurst, and Hellige (1984) found no evidence of sex differences in the mental solution of easy and difficult block designs. Curiously, they observed more left-hemisphere involvement in these tasks. In their second experiment, Kee et al. did not analyze for sex differences, but they found right-hemisphere lateralization when subjects manually solved the block designs.

In the face of these perplexing findings, we decided to partially replicate and extend both the Hellige and Longstreth (1981) and Kee et al. (1984) studies in an attempt to determine the reliability of these findings. Furthermore, we attempted to establish the role of verbal mediation in visuospatial processing in males and females. Using two difficulty levels of block designs in a dual-task paradigm, we hypothesized that, in the easy condition, both sexes would show right-hemisphere control, indexed by interference with left-hand tapping rates in both males and females; in the difficult condition, males would display asymmetrical declines in left-hand tapping, and females would display symmetrical declines in both right- and lefthand tapping. This pattern would suggest right lateralization in males and bilateralization in females. It was also hypothesized that if the dual-task paradigm did not result in hand-tapping interference, then an interference effect would be observed in time taken to manually solve the block designs. We further hypothesized that females use verbal mediation as a strategy in visuospatial tasks of this kind, and that simultaneous vocalization would selectively interfere with either the time taken to solve block designs or in the rate of vocalization in females only. Nichelli, Manni, and Faglioni (1983) and McGlone and Kertesz 
(1973) have proposed a verbal mediation strategy in females to account for sex differences in spatial processing. Since females are hypothesized to be more bilateralized in processing spatial problems, vocalization should activate the left hemisphere and selectively interfere with spatial processing in females.

\section{METHOD}

\section{Subjects}

Fifty undergraduate students participated in the study, 21 males and 29 females. All subjects were right-handed as assessed by the Edinburgh Handedness Inventory (Oldfield, 1971).

\begin{abstract}
Apparatus
The apparatus used for finger tapping consisted of a telegraph key mounted on a wooden base. The key was $2.7 \mathrm{~cm}$ in diameter. The key was positioned on a table in front of the subject's shoulder and on the side of the hand used during tapping so that the forearm rested on the tabletop. The number of taps was recorded on a BSR Foeringer counter panel (Model CP-901), which was activated by a Tenor timer (Model TI-902). The timer was set for $15 \mathrm{sec}$. The counter was read at the end of each 15-sec trial and reset. The spatial problems consisted of the Wechsler Intelligence Scale for Children-Revised block designs 6, 7 , 8,10 , and 11, and designs $4,5,6,7$, and 8 from the Wechsler Adult Intelligence Scale-Revised.
\end{abstract}

\section{Experimental Design}

The independent variables examined in Phase 1 of this study were as follows: two within-subjects variables of hand used (right or left) and task (concurrent tapping with an easy block design, difficult design, or tapping alone) and one between-subjects variable of sex. The dependent measure for this phase of the study was the number of taps produced in a 15 -sec trial. Thus, the design is a $2 \times 3 \times 2$ mixed-model ANOVA. To determine whether there were any trade-off effects, the time taken to solve the block designs in the baseline and dual-task conditions was compared using a factorial design ANOVA.

In the second phase of the study, the independent variables were the within-subjects variables of hand and task (easy block design, difficult design, vocalization with easy design, and vocalization with difficult design) and the between-subjects variable of sex. The dependent variable was the time taken to complete a block design. The analysis used was a mixedmodel ANOVA design. Trade-off effects were analyzed with a similar ANOVA, with the number of vocalizations as the dependent variable.

\section{Procedure}

Each subject was tested individually following determination of righthandedness. Subjects were instructed how to tap on the telegraph key with the index finger while fixating on a blank piece of white paper taped to the wall in front of them. In Phase 1, there were 10 experimental conditions involving tapping and/or performing a spatial task. Each subject completed one 15-sec trial in each of the following conditions: (1) LC, left-hand tapping alone; (2) RC, right-hand tapping alone; (3) LE, manual solution of easy block design with left hand; (4) RE, manual solution of block design with right hand; (5) LD, manual solution of difficult design with left hand; (6) RD, manual solution of difficult design with right hand; (7) LER, left-hand tapping and manual solution of easy design using right hand; (8) REL, right-hand tapping and manual solution of easy design using left hand; (9) LDR, left-hand tapping and manual solution of difficult design using right hand; and (10) RDL, right-hand tapping and manual solution of difficult design using left hand.

The easy block designs consisted of Designs 4 and 5 from the WAIS-R, and 6,7 , and 8 from the WISC-R. The difficult block designs were designs 6, 7, and 8 from the WAIS-R, and Designs 10 and 11 from the WISC-R. Presentation of the designs was randomized and counterbalanced so that an equal number of subjects received each condition of designs in every possible starting position. This procedure was used to control for practice and order effects in solving the easy and difficult block designs.
Each subject was given a brief practice trial in tapping the telegraph key. Before each trial, the subject was told what each of the following tasks would involve. Subjects were told that the purpose of the experiment was to see how rapidly they could perform two simultaneous tasks, and that they were to tap as fast as they could and/or solve a spatial problem as quickly as possible when the experimenter said "Go," until the command "Stop" was given. Each subject was also instructed to continue to check the block design for accuracy if the design was completed before the time trial was over. Each subject was then given the 10 trial conditions.

In Phase 2 of the experiment, subjects completed the five conditions of verbal task alone and verbal tasks concurrently with the two levels of spatial tasks (easy and difficult). The results from Phase 1 baselines were used. The verbal task involved repetition of the phrase "cat, dog, horse" as rapidly as possible during a 15-sec trial. The experimental conditions were as follows: (1) VC, vocalization alone; (2) VEL, vocalization and easy block design with left hand; (3) VER, vocalization and easy block design with right hand; (4) VDL, vocalization and difficult design with left hand; and (5) VDR, vocalization and difficult design with right hand. Vocalizations of each subject were tape-recorded during the trials and independently counted by two judges after the experiment was completed.

\section{RESULTS}

Change scores were computed for each dependent variable by subtracting the dual-task score from the baseline score:

\section{Change Score $=$ Baseline Score - Dual Task Score}

A mixed-model ANOVA was used to analyze tapping rates, with sex as the between-subjects variable and tapping hand (right vs. left) and difficulty level (easy vs. difficult) as the within-subjects variables. No significant differences in change scores for tapping rates were found between sex, hand used, and task difficulty level.

A similar ANOVA was used to analyze trade-off effects of time taken to complete the block designs. There was an overall main trade-off effect of time to solution and hand used $[F(1,46)=4.30, p<.05]$. Both males and females took significantly longer to complete the two levels of block designs when tapping with the right hand than when tapping with the left. The mean change score for time to block design solution was $4.85 \mathrm{sec}$ with lefthand tapping, and $-11.42 \mathrm{sec}$ with right-hand tapping. Time to solution significantly declined during left-hand tapping from baseline to dual task for both males and females $[t(50)=3.65, p<.05]$. In the right-hand tapping condition, time taken to solve the designs significantly increased from baseline to dual task for both males and females $[t(50)=8.52, p=.001]$.

In Phase 2 of this study, we attempted to assess the change in block design performance associated with induced interference of possible verbal mediation strategies. A mixed-model ANOVA was used to examine the change in time to completion of the block designs during concurrent vocalization, with sex as the between-subjects variable and hand used in block design manipulation and task difficulty as within-subjects variables. There was a significant main effect for $\operatorname{sex}[F(1,48)=7.59, p<.01]$. Females displayed more interference than did males in block design solution during concurrent vocalization. The 
mean change score in time taken to solve the block designs was $-4.11 \mathrm{sec}$ for females and $11.86 \mathrm{sec}$ for males.

Overall, females took significantly longer to solve the block designs during simultaneous vocalization than did males. The increase in time of design solution for females from baseline to dual task was not significant $[t(42)=$ .34]. The observed decrease in time to solution from baseline to dual task for males was significant $[t(42)=18.25$, $p<.001]$.

A significant sex $\times$ difficulty interaction was also found $[F(1,48)=9.70, p<.01]$. Post hoc analysis revealed that females showed significantly greater interference effects when solving the difficult designs. For the easy block designs, the mean change score for time to solution in the dual-task condition was $-.56 \mathrm{sec}$ for females and $-3.30 \mathrm{sec}$ for males. The mean time to solution scores were not significantly different from baseline. For the difficult designs, the mean change score for solution time was -8.8 for females and 27.36 for males during concurrent vocalization. For both males and females, the mean time scores were significantly different from baseline $[t(42)=2.86, p<.05$, and $t(42)=13.93$, $p<.001$, respectively]. Females took significantly longer to solve the designs in the dual-task condition, whereas males took significantly less time in the dual task compared to baseline measures.

A similar mixed-model ANOVA was used to analyze the change in the number of words uttered during concurrent block design solution to determine possible tradeoff effects. There was a main trade-off effect for difficulty level $[F(1,48)=4.43, p<.05]$, suggesting that across sex and hand used there was a greater reduction in vocalization under the difficult design condition. The mean change score for number of words uttered was 2.71 during the easy design condition and 8.49 during the difficult design condition. The mean change in number of vocalizations significantly decreased in the difficult design condition $[t(42)=6.56, p<.001]$.

\section{DISCUSSION}

Our first hypothesis of selective interference in tapping as a function of difficulty of spatial tasks was not supported. We found no evidence of differential laterality in finger tapping either within or between sexes in the dual-task paradigm, regardless of the difficulty level of visuospatial processing; that is, spatial task difficulty had no effect on rate of tapping in males and females, and both right- and left-hand tapping during the concurrent task were not significantly different from baseline rates.

Unlike Kee et al. (1984) and Hellige and Longstreth (1981), we found no evidence of right-cerebral lateralization for spatial processing using finger tapping as the index of cerebral control. However, when we analyzed for trade-off effects in the amount of time taken to complete the spatial tasks, we found a significant interference effect.

All subjects took longer to complete the block designs (collapsed across difficulty level) only when simultaneously tapping with the right hand and manipulating the block designs with the left. When subjects used their right hands to solve the block designs, the time taken to solution significantly declined from baseline to dual task. This effect was observed for both males and females, indicating that the pattern of cerebral organization for this type of spatial processing is similar in both males and females. These data suggest that when the left hand is used in manipulospatial processing, the combined demands of right-hemisphere motor control of the hand and spatial processing result in less efficient spatial processing (longer solution times) for males and females. During right-hand manipulospatial processing and left-hand tapping, there appears to have been a facilitative effect in spatial processing speed for both sexes. Since motor control of the right hand and spatial processing are hemispherically separated, the functional distance between lefthand tapping control and spatial processing may be great enough to prevent interference, but close enough to facilitate manipulospatial processing. Since no laterality effects were observed in right-hand and left-hand tapping, it is not possible to identify any lateralization patterns for manipulospatial processing of block designs in either males or females, except to say that both show the same interference patterns.

It is interesting to note that most investigators using the dual-task paradigm of simultaneous motor tasks and spatial processing report unidirectional interference effects on motor performance only. On the other hand, most of these studies do not include an analysis of possible trade-off effects in the temporal aspects of spatial processing. According to Kinsbourne's (1981) functional distance hypothesis, decrements in performance may be observed in either task. Since Kee et al. (1984) and Hellige and Longstreth (1981) report no such analysis, it is difficult to clearly interpret their results.

In Phase 2 , it was observed that during concurrent vocalization females showed greater interference effects in solving the block designs than did males. As predicted, females took significantly longer in block design solution in this dual-task condition than did the males, regardless of hand used in manipulospatial processing. For males, concurrent vocalization resulted in an overall decrease in time taken to complete the designs, and this decrease was significant from baseline time scores. The females showed interference effects when solving the difficult designs, suggesting that verbal mediation may be a factor at this level of spatial processing in females. Simultaneous vocalization appeared to have disrupted the more difficult manipulospatial processing in females and to have facilitated it in males, regardless of hand used. The time score changes for both females and males in the dual task were significantly different from baseline measures.

The trade-off effects in Phase 2 were limited to difficulty level of the block designs. That is, there was a significant reduction in the number of words uttered during the difficult dual-task condition, whereas there were no differential sex effects in number of words spoken during manipulospatial processing. Likewise, no trade-off effects were observed as a function of hand used. These data indicate that the verbal interference effect shown in the female subjects was not a function of differential attention effects. Rather, they suggest that left-cerebral activation elicited by vocalization results in less efficient manipulospatial processing in females. Unsupported by any evidence of laterality effects in Phase 1, the data from Phase 2 only weakly support the hypothesis of symmetrical organization for spatial processing in females. Overall, we are unable to report any evidence of either differential lateralization patterns in males or females or support for right-cerebral control of the type of manipulospatial processing used in this study. The major findings of this study revolve around the verbal interference effects found in females and the evidence of their use of a verbal mediation strategy in manipulospatial processing of this kind.

The underlying mechanisms of the differential performance of males and females on spatial tasks remain elusive. Our data weakly suggest the possibility of female bilateralization of visuospatial processing. The clarification of brain-behavior relationships in spatial abilities will require a more careful delineation of the cognitive components represented in spatial processing, and which of these differentiate the sexes in terms of performance efficiency. Perhaps an examination of extreme group performance in males and females may shed some light on the biological basis of such differences.

\section{REFERENCES}

Buffery, A. W. H., \& Gray, J. A. (1972). Sex differences in the development of spatial and linguistic skills. In C. Ounsted \& D. C. Taylor (Eds.), Gender differences: Their ontogeny and significance. London: Churchill.

DALBY, J. T. (1980). Hemispheric timesharing: Verbal and spatial loading with concurrent unimanual activity. Cortex, 16, 567-573.

Hellige, J. B., \& LoNGSTRETH, L. E. (1981). Effects of concurrent 
hemispheric-specific activity on unimanual tapping rate. Neuropsychologia, 19, 395-405.

Kee, D. W., Bathhurst, K., \& Hellige, J. B. (1984). Lateralized interference in finger tapping: Assessment of block design activities. Neuropsychologia, 22, 197-203.

KINSBOURNE, M. (1981). The development of cerebral dominance. In S. B. Filskov \& T. J. Bolls (Eds.), Handbook of clinical neuropsychology. New York: Wiley.

LEVY, J. (1972). Lateral specialization of the human brain: Behavioral manifestations and possible evolutionary basis. In J. A. Kiger (Ed.), The biology of behavior. Corvallis: Oregon University Press.

McGlone, J., \& KerTeSz, A. (1973). Sex differences in cerebral processing of visuospatial tasks. Cortex, 9, 313-320.
Nichelli, P., Manni, A., \& Faglioni, P. (1983). Relationships between speed, accuracy of performance, and hemispheric superiorities for visuo-spatial pattern processing in the two sexes. Neuropsychologia, 21, 625-632.

OLDFIELD, R. C. (1971). The assessment and analysis of handedness: Edinburgh Inventory. Neuropsychologia, 9, 97-113.

WolfF, P. H., HURWITz, I., \& Moss, H. (1977). Serial organization of motor skills in left and right-handed adults. Neuropsychologia, 15, 536-546.

(Manuscript received for publication January 12, 1987.) 